\title{
A note on $q$-analogue of Hermite-poly-Bernoulli numbers and polynomials
}

\author{
Waseem A. Khan, Idrees A. Khan, Musharraf Ali
}

\begin{abstract}
In this paper, we introduce the Hermite-based poly-Bernoulli numbers and polynomials with $q$-parameter and give some of their basic properties including not only addition property, but also derivative properties and integral representations. We also define the Hermitebased $\lambda$-Stirling polynomials of the second kind and then provide some relations, identities of these polynomials related to the Stirling numbers of the second kind. We derive some symmetric identities for these families of special functions by applying the generating functions.
\end{abstract}

\section{INTRODUCTION}

Throughout this paper, we use the following notations, $\mathbb{N}=\{1,2,3, \ldots\}$ denotes the set of natural numbers, $\mathbb{N}_{0}=\{1,2,3, \ldots\}$ denotes the set of non negative integer, $\mathbb{Z}$ denotes the set of integers and $\mathbb{C}$ denotes the set of complex numbers respectively.

The 2-variable Kampé de Fériet generalization of the Hermite polynomials [3] and [8] are defined by

$$
H_{n}(x, y)=n ! \sum_{r=0}^{\left[\frac{n}{2}\right]} \frac{y^{r} x^{n-2 r}}{r !(n-2 r) !} .
$$

These polynomials are usually defined by the generating function:

$$
e^{x t+y t^{2}}=\sum_{n=0}^{\infty} H_{n}(x, y) \frac{t^{n}}{n !},
$$

and reduce to the ordinary Hermite polynomials $H_{n}(x)$ (see [1]), when $y=$ -1 and $x$ is replaced by $2 x$.

2010 Mathematics Subject Classification. Primary: 11B68, 11B73, 11B75, 33C45.

Key words and phrases. Hermite polynomials, $q$-analogue of poly-Bernoulli polynomials, $q$-analogue of Hermite poly-Bernoulli polynomials, Stirling numbers of the second kind, $q$-polylogarithm function, Symmetric identities.

Full paper. Received 4 October 2018, revised 9 May 2019, accepted 24 June 2019, available online 1 October 2019. 
As is well known, the Bernoulli polynomials are defined by the generating function as

$$
\left(\frac{t}{e^{t}-1}\right) e^{x t}=\sum_{n=0}^{\infty} B_{n}(x) \frac{t^{n}}{n !}, \quad(\text { see }[1-27]) .
$$

When $x=0, B_{n}=B_{n}(0)$ are called the Bernoulli numbers.

From (1.2), we have

$$
B_{n}(x)=\sum_{m=0}^{n}\left(\begin{array}{c}
n \\
m
\end{array}\right) B_{n-m} x^{m} .
$$

The classical polylogarithm function $\operatorname{Li}_{k}(z)$ is

$$
\operatorname{Li}_{k}(z)=\sum_{m=1}^{\infty} \frac{z^{m}}{m^{k}}, \quad(k \in \mathbb{Z}), \quad(\text { see }[10,11,12]) .
$$

So, for $k \leq 1$,

$$
\mathrm{Li}_{k}(z)=-\ln (1-z), \quad \operatorname{Li}_{0}(z)=\frac{z}{1-z}, \quad \operatorname{Li}_{-1}(z)=\frac{z}{(1-z)^{2}}, \ldots
$$

The poly-Bernoulli polynomials are given by

$$
\frac{\operatorname{Li}_{k}\left(1-e^{-t}\right)}{e^{t}-1} e^{x t}=\sum_{n=0}^{\infty} B_{n}^{(k)}(x) \frac{t^{n}}{n !}, \quad(\text { see }[10,15-19]) .
$$

For $k=1$, we have

$$
\frac{\operatorname{Li}_{1}\left(1-e^{-t}\right)}{e^{t}-1} e^{x t}=\frac{t}{e^{t}-1} e^{x t}=\sum_{n=0}^{\infty} B_{n}(x) \frac{t^{n}}{n !} .
$$

From (1.2) and (1.3), we obtain

$$
B_{n}^{(1)}(x)=B_{n}(x), \quad(n \geq 0) .
$$

Very recently, Khan et al. [14] introduced the Hermite poly-Bernoulli polynomials of two variables ${ }_{H} B_{n}^{(k)}(x, y)$ defined by

$$
\left(\frac{\operatorname{Li}_{k}\left(1-e^{-t}\right)}{e^{t}-1}\right) e^{x t+y t^{2}}=\sum_{n=0}^{\infty}{ }_{H} B_{n}^{(k)}(x, y) \frac{t^{n}}{n !},
$$

which is essentially a generalization of Bernoulli numbers, Bernoulli polynomials, Hermite polynomials and Hermite-Bernoulli polynomials ${ }_{H} B_{n}(x, y)$ introduced by Dattoli et al. [8, p.386(1.6)] in the form:

$$
\left(\frac{t}{e^{t}-1}\right) e^{x t+y t^{2}}=\sum_{n=0}^{\infty}{ }_{H} B_{n}(x, y) \frac{t^{n}}{n !} .
$$

The Stirling number of the first kind is given by

$$
(x)_{n}=x(x-1) \cdots(x-n+1)=\sum_{l=0}^{n} S_{1}(n, l) x^{l}, \quad(n \geq 0), \quad(\text { see }[4,9,26])
$$


and the Stirling number of the second kind is defined by generating function

$$
\left(e^{t}-1\right)^{n}=n ! \sum_{l=n}^{\infty} S_{2}(l, n) \frac{t^{l}}{l !} .
$$

This paper organized as follows. In Section 2, we introduce a new class of Hermite poly-Bernoulli numbers and polynomials with $q$-parameter. In Section 3, we establish some identities of these polynomials. In Section 4, we derive some properties of the Stirling numbers of the second kind. In Section 5, we derive symmetric identities for these generalized polynomials by using different analytical means on their respective generating functions.

\section{A note on $q$-Analogue of Hermite-Poly-Bernoulli numbers AND POLYNOMIALS}

In this section, we define a $q$-analogue of Hermite-poly-Bernoulli numbers and polynomials and its properties.

Definition 2.1. For $n \geq 0, n, k \in \mathbb{Z}, 0 \leq q<1$, we introduce a $q$-analogue of Hermite-poly-Bernoulli polynomials by means of the following generating function:

$$
\frac{\operatorname{Li}_{k, q}\left(1-e^{-t}\right)}{e^{t}-1} e^{x t+y t^{2}}=\sum_{n=0}^{\infty}{ }_{H} B_{n, q}^{(k)}(x, y) \frac{t^{n}}{n !},
$$

where $\operatorname{Li}_{k, q}(t)=\sum_{n=0}^{\infty} \frac{t^{n}}{[n]_{q}^{k !}}$ is the $k$-th $q$-polylogarithm function (see $[4,6,23]$ ).

When $x=y=0, B_{n, q}^{(k)}={ }_{H} B_{n, q}^{(k)}(0,0)$ are called a $q$-analogue polyBernoulli numbers.

Remark 2.1. For $y=0$ in (2.1), the result reduces to the known result of Hwang et al. [9] as follows:

$$
\frac{\operatorname{Li}_{k, q}\left(1-e^{-t}\right)}{e^{t}-1} e^{x t}=\sum_{n=0}^{\infty} B_{n, q}^{(k)}(x, y) \frac{t^{n}}{n !} .
$$

From (2.1), we have

$$
\lim _{q \longrightarrow 1} \frac{\operatorname{Li}_{1}\left(1-e^{-t}\right)}{e^{t}-1} e^{x t+y t^{2}}=\sum_{n=0}^{\infty} H_{n} B_{n}(x, y) \frac{t^{n}}{n !} .
$$

Thus by (2.1) and (2.2), we get

$$
{ }_{H} B_{n}^{(k)}(x, y)={ }_{H} B_{n}(x, y) \quad(n \geq 0), \quad(\text { see }[21,22]) .
$$

Theorem 2.1. (Addition Property), we have

$$
{ }_{H} B_{n, q}^{(k)}\left(x_{1}+x_{2}, y_{1}+y_{2}\right)=\sum_{m=0}^{n} \text { binomnm }_{H} B_{n-m, q}^{(k)}\left(x_{1}, y_{1}\right) H_{m}\left(x_{2}, y_{2}\right) \text {. }
$$


Proof. Consider (2.1), we have

$$
\begin{gathered}
\sum_{n=0}^{\infty}{ }_{H} B_{n, q}^{(k)}\left(x_{1}+x_{2}, y_{1}+y_{2}\right) \frac{t^{n}}{n !}=\frac{\operatorname{Li}_{k, q}\left(1-e^{-t}\right)}{e^{t}-1} e^{\left(x_{1}+x_{2}\right) t+\left(y_{1}+y_{2}\right) t^{2}} \\
=\sum_{n=0}^{\infty}{ }_{H} B_{n, q}^{(k)}\left(x_{1}, y_{1}\right) \frac{t^{n}}{n !} \sum_{m=0}^{\infty} H_{m}\left(x_{2}, y_{2}\right) \frac{t^{m}}{m !} \\
=\sum_{n=0}^{\infty}\left(\sum_{m=0}^{n}\left(\begin{array}{c}
n \\
m
\end{array}\right){ }_{H} B_{n-m, q}^{(k)}\left(x_{1}, y_{1}\right) H_{m}\left(x_{2}, y_{2}\right)\right) \frac{t^{n}}{n !} .
\end{gathered}
$$

Equating the coefficients of $\frac{t^{n}}{n !}$ in both sides, we get (2.3).

Theorem 2.2. (Derivative Properties) Each of the following formula holds true:

and

$$
\frac{\delta_{H} B_{n, q}^{(k)}(x, y)}{\delta x}=n_{H} B_{n-1, q}^{(k)}(x, y)
$$

$$
\frac{\delta_{H} B_{n, q}^{(k)}(x, y)}{\delta y}=n(n-1)_{H} B_{n-2, q}^{(k)}(x, y) .
$$

Proof. The proof follows from (2.1). So we omit them.

Theorem 2.3. (Integral Representation) The following formula holds true:

$$
\int_{r}^{s}{ }_{H} B_{n, q}^{(k)}(x, y) d x=\frac{{ }_{H} B_{n+1, q}^{(k)}(s, y)-{ }_{H} B_{n+1, q}^{(k)}(r, y)}{n+1}
$$

and

$$
\int_{\lambda}^{\mu}{ }_{H} B_{n, q}^{(k)}(x, y) d y=\frac{{ }_{H} B_{n+2, q}^{(k)}(x, \mu)-{ }_{H} B_{n+2, q}^{(k)}(x, \lambda)}{(n+1)^{2}} .
$$

Proof. Using the derivative properties of ${ }_{H} B_{n, q}^{(k)}(x, y)$ given in Theorem 2.2, we easily get the asserted results. So we omit them.

Theorem 2.4. The following formula holds true:

$$
{ }_{H} B_{n, q}^{(k)}(x, y)=\sum_{m=0}^{\left[\frac{n}{2}\right]} B_{n-m, q}^{(k)}(x) y^{m} \frac{n !}{m !(n-2 m) !} .
$$

Proof. By (2.1), we have

$$
\begin{aligned}
\sum_{n=0}^{\infty}{ }_{H} B_{n, q}^{(k)}(x, y) \frac{t^{n}}{n !} & =\frac{\operatorname{Li}_{k, q}\left(1-e^{-t}\right)}{e^{t}-1} e^{x t+y t^{2}} \\
& =\sum_{n=0}^{\infty} B_{n, q}^{(k)}(x) \frac{t^{n}}{n !} \sum_{m=0}^{\infty} \frac{y^{m} t^{2 m}}{m !}
\end{aligned}
$$




$$
=\sum_{n=0}^{\infty}\left(\sum_{m=0}^{\left[\frac{n}{2}\right]} B_{n-m, q}^{(k)}(x) y^{m}\right) \frac{t^{n}}{m !(n-2 m) !},
$$

which implies the desired result (2.4).

\section{Main Results}

In this section, we establish some properties of $q$-analogue of Hermitepoly-Bernoulli polynomials by using generating function.

Theorem 3.1. For $n \geq 0, n, k \in \mathbb{Z}, 0 \leq q<1$, we have

$$
{ }_{H} B_{n, q}^{(k)}(x, y)=\sum_{m=0}^{n}\left(\begin{array}{c}
n \\
m
\end{array}\right) B_{n-m, q}^{(k)} H_{m}(x, y) .
$$

Proof. By using (2.1) and (1.1), we arrive at the desired result (3.1).

Theorem 3.2. For $n \geq 0$, we have

$$
{ }_{H} B_{n, 1}^{(2)}(x, y)=\sum_{m=0}^{n}\left(\begin{array}{c}
n \\
m
\end{array}\right) \frac{B_{m} m !}{m+1}{ }_{H} B_{n-m}(x, y) .
$$

Proof. Consider (2.1), we have

$$
\begin{gathered}
\sum_{n=0}^{\infty} H B_{n, 1}^{(k)}(x, y) \frac{t^{n}}{n !}=\frac{\operatorname{Li}_{k, 1}\left(1-e^{-t}\right)}{e^{t}-1} e^{x t+y t^{2}} \\
=\frac{e^{x t+y t^{2}}}{e^{t}-1} \underbrace{\int_{0}^{t} \frac{1}{e^{z}-1} \int_{0}^{t} \frac{1}{e^{z}-1} \cdots \frac{1}{e^{z}-1}}_{(k-1)-\text { times }} \int_{0}^{t} \frac{z}{e^{z}-1} d z \cdots d z .
\end{gathered}
$$

In particular for $k=2$, we have

$$
\begin{aligned}
\sum_{n=0}^{\infty}{ }_{H} B_{n}^{(2)}(x, y) \frac{t^{n}}{n !} & =\frac{e^{x t+y t^{2}}}{e^{t}-1} \int_{0}^{t} \frac{z}{e^{z}-1} d z \\
& =\left(\sum_{m=0}^{\infty} \frac{t^{m} B_{m}}{m+1}\right) \frac{t}{e^{t}-1} e^{x t+y t^{2}} \\
& =\left(\sum_{m=0}^{\infty} \frac{B_{m} m !}{m+1} \frac{t^{m}}{m !}\right)\left(\sum_{n=0}^{\infty} H B_{n}(x, y) \frac{t^{n}}{n !}\right) .
\end{aligned}
$$

Replacing $n$ by $n-m$ in R.H.S. of above equation, we have

$$
=\sum_{n=0}^{\infty} \sum_{m=0}^{n}\left(\begin{array}{c}
n \\
m
\end{array}\right) \frac{B_{m} m !}{m+1}{ }_{H} B_{n-m}(x, y) \frac{t^{n}}{n !} .
$$

On comparing the coefficients of $\frac{t^{n}}{n !}$ on both sides of the above equation, we get the result (3.2). 
Theorem 3.3. For $n \geq 0, n, k \in \mathbb{Z}, 0 \leq q<1$, we have

$$
{ }_{H} B_{n, q}^{(k)}(x, y)=\sum_{l=0}^{\infty} \frac{1}{[l+1]_{q}^{k}} \sum_{a=0}^{l+1}\left(\begin{array}{c}
l+1 \\
a
\end{array}\right)(-1)^{a} \frac{H B_{n+1}(x-a, y)}{n+1} .
$$

Proof. From equation (2.1), we have

$$
\sum_{n=0}^{\infty} H_{n, q}^{(k)}(x, y) \frac{t^{n}}{n !}=\frac{\operatorname{Li}_{k, q}\left(1-e^{-t}\right)}{e^{t}-1} e^{x t+y t^{2}}
$$

Now

$$
\begin{gathered}
\frac{\operatorname{Li}_{k, q}\left(1-e^{-t}\right)}{e^{t}-1} e^{x t+y t^{2}}=\sum_{l=1}^{\infty} \frac{\left(1-e^{-t}\right)^{l}}{[l]_{q}^{k}} \frac{e^{x t+y t^{2}}}{e^{t}-1} \\
=\sum_{n=0}^{\infty} \frac{1}{[l]_{q}^{k}} \sum_{a=0}^{l+1}\left(\begin{array}{c}
l+1 \\
a
\end{array}\right)(-1)^{a} \frac{e^{(x-a) t+y t^{2}}}{e^{t}-1} \\
=\sum_{n=0}^{\infty}\left(\sum_{l=0}^{\infty} \frac{1}{[l+1]_{q}^{k}} \sum_{a=0}^{l+1}\left(\begin{array}{c}
l+1 \\
a
\end{array}\right)(-1)^{a} \frac{H B_{n+1}(x-a, y)}{n+1}\right) \frac{t^{n}}{n !} .
\end{gathered}
$$

Comparing the coefficients of $\frac{t^{n}}{n !}$ on both sides, we get the result (3.3).

Theorem 3.4. For $n \geq 0, n, k \in \mathbb{Z}, 0 \leq q<1$, we have

$$
{ }_{H} B_{n, q}^{(k)}(x, y)=\sum_{l=0}^{\infty} \sum_{m=0}^{l} \sum_{a=0}^{m+1}\left(\begin{array}{c}
m+1 \\
a
\end{array}\right)(-1)^{a} \frac{H_{n}(l-m-a+x, y)}{[m+1]_{q}^{k}}
$$

Proof. From equation (2.1), we have

$$
\begin{aligned}
& \sum_{n=0}^{\infty} H_{n, q}^{(k)}(x, y) \frac{t^{n}}{n !}=\frac{\operatorname{Li}_{k, q}\left(1-e^{-t}\right)}{e^{t}-1} e^{x t+y t^{2}} \\
& =\left(\sum_{m=0}^{\infty} e^{m t}\right)\left(\sum_{l=0}^{\infty} \frac{\left(1-e^{-t}\right)^{l+1}}{[l+1]_{q}^{k}}\right) e^{x t+y t^{2}} \\
& =\left(\sum_{l=0}^{\infty} \sum_{m=0}^{l} \frac{e^{(l-m) t}}{[m+1]_{q}^{k}}\right)\left(\sum_{a=0}^{m+1}\left(\begin{array}{c}
m+1 \\
a
\end{array}\right)(-1)^{a} e^{(x-a) t+y t^{2}}\right) \\
& =\sum_{l=0}^{\infty} \sum_{m=0}^{l} \sum_{a=0}^{m+1}\left(\begin{array}{c}
m+1 \\
a
\end{array}\right)(-1)^{a} \frac{e^{(l-m-1+x) t+y t^{2}}}{[m+1]_{q}^{k}} \\
& \sum_{n=0}^{\infty} H B_{n, q}^{(k)}(x, y) \frac{t^{n}}{n !} \\
& =\sum_{n=0}^{\infty} \sum_{l=0}^{\infty} \sum_{m=0}^{l} \sum_{a=0}^{m+1}\left(\begin{array}{c}
m+1 \\
a
\end{array}\right)(-1)^{a} \frac{H_{n}(l-m-a+x, y)}{[m+1]_{q}^{k}} \frac{t^{n}}{n !} .
\end{aligned}
$$

Equating the coefficients of $\frac{t^{n}}{n !}$ in both sides, we get (3.4). 
Theorem 3.5. The following implicit summation formulae for Hermite poly-Bernoulli polynomials ${ }_{H} B_{n, q}^{(k)}(x, y)$ holds true:

$$
{ }_{H} B_{l+p, q}^{(k)}(z, y)=\sum_{m, n=0}^{l, p}\left(\begin{array}{c}
l \\
m
\end{array}\right)\left(\begin{array}{l}
p \\
n
\end{array}\right)(z-x)^{m+n}{ }_{H} B_{l+p-m-n, q}^{(k)}(x, y) .
$$

Proof. We replace $t$ by $t+u$ and rewrite the generating function (2.1) as

$$
\frac{\operatorname{Li}_{k, q}\left(1-(e)^{-(t+u)}\right)}{e^{t+u}-1} e^{y(t+u)^{2}}=e^{-x(t+u)} \sum_{l, p=0}^{\infty}{ }_{H} B_{l+p, q}^{(k)}(x, y) \frac{t^{l}}{l !} \frac{u^{p}}{p !} .
$$

Replacing $x$ by $z$ in the above equation and equating the resulting equation to the above equation, we get

$$
e^{(z-x)(t+u)} \sum_{m, l=0}^{\infty} H_{l+p, q}^{(k)}(x, y) \frac{t^{l}}{l !} \frac{u^{p}}{p !}=\sum_{l, p=0}^{\infty} H_{l+p, q}^{(k)}(z, y) \frac{t^{l}}{l !} \frac{u^{p}}{p !} .
$$

On expanding exponential function, (3.3) gives

$$
\sum_{N=0}^{\infty} \frac{[(z-x)(t+u)]^{N}}{N !} \sum_{l, p=0}^{\infty}{ }_{H} B_{l+p, q}^{(k)}(x, y) \frac{t^{l}}{l !} \frac{u^{p}}{p !}=\sum_{l, p=0}^{\infty}{ }_{H} B_{l+p, q}^{(k)}(z, y) \frac{t^{l}}{l !} \frac{u^{p}}{p !},
$$

which on using formula [27, p.52(2)]

$$
\sum_{N=0}^{\infty} f(N) \frac{(x+y)^{N}}{N !}=\sum_{n, m=0}^{\infty} f(n+m) \frac{x^{n}}{n !} \frac{y^{m}}{m !},
$$

in the left hand side becomes

$$
\begin{gathered}
\sum_{m, n=0}^{\infty} \frac{(z-x)^{m+n} t^{m} u^{n}}{m ! n !} \sum_{l, p=0}^{\infty}{ }_{H} B_{l+p, q}^{(k)}(x, y) \frac{t^{l}}{l !} \frac{u^{p}}{p !}= \\
=\sum_{l, p=0}^{\infty} H_{l+p, q} B_{l, ~(k)}(z, y) \frac{t^{l}}{l !} \frac{u^{p}}{p !} .
\end{gathered}
$$

Now replacing $l$ by $l-m, p$ by $p-n$ and using the lemma [27, p.100(1)] in the left hand side of (3.5), we get

$$
\begin{gathered}
\sum_{m, n=0}^{\infty} \sum_{l, p=0}^{\infty} \frac{(z-x)^{m+n}}{m ! n !}{ }_{H} B_{l+p-m-n, q}^{(k)}(x, y) \frac{t^{l}}{(l-m) !} \frac{u^{p}}{(p-n) !}= \\
=\sum_{l, p=0}^{\infty} H_{l+p, q} B^{(k)}(z, y) \frac{t^{l}}{l !} \frac{u^{p}}{p !} .
\end{gathered}
$$

Finally on equating the coefficients of the like powers of $t$ and $u$ in the above equation, we get the required result. 
Remark 3.1. By taking $l=0$ in Equation (3.1), we immediately deduce the following result.

Corollary 3.1. The following implicit summation formula for Hermite polyBernoulli polynomials ${ }_{H} B_{n, q}^{(k)}(z, y)$ holds true:

$$
{ }_{H} B_{p, q}^{(k)}(z, y)=\sum_{n=0}^{p}\left(\begin{array}{l}
p \\
n
\end{array}\right)(z-x)^{n}{ }_{H} B_{p-n, q}^{(k)}(x, y) .
$$

Remark 3.2. On replacing $z$ by $z+x$ and setting $y=0$ in Theorem 3.1, we get the following result involving poly-Bernoulli polynomials of one variable.

$$
{ }_{H} B_{l+p, q}^{(k)}(z+x)=\sum_{m, n=0}^{l, p}\left(\begin{array}{c}
l \\
m
\end{array}\right)\left(\begin{array}{l}
p \\
n
\end{array}\right) z^{m+n} B_{l+p-m-n, q}^{(k)}(x),
$$

where as, by setting $z=0$ in Theorem 3.1, we get another result involving $q$-poly-Bernoulli polynomials of one and two variables.

$$
B_{l+p, q}^{(k)}(y)=\sum_{m, n=0}^{l, p}\left(\begin{array}{c}
l \\
m
\end{array}\right)\left(\begin{array}{l}
p \\
n
\end{array}\right)(-x)^{m+n}{ }_{H} B_{l+p-m-n, q}^{(k)}(x, y) .
$$

Remark 3.3. Along with the above results we will exploit extended forms of $q$-poly-Bernoulli polynomials $B_{l+p, q}^{(k)}(z)$ by setting $y=0$ in Theorem 3.1 to get

$$
B_{l+p, q}^{(k)}(z)=\sum_{m, n=0}^{l, p}\left(\begin{array}{c}
l \\
m
\end{array}\right)\left(\begin{array}{l}
p \\
n
\end{array}\right)(z-x)^{n+m} B_{l+p-m-n, q}^{(k)}(x) .
$$

4. The $q$-Analogue of Hermite-Based Stirling POLYNOMIALS OF THE SECOND KIND

In this section, we introduce $q$-analogue of Hermite-based Stirling polynomials of the second kind is defined by

$$
\frac{\left(e^{t}-1\right)^{m}}{m !} e^{x t+y t^{2}}=\sum_{n=0}^{\infty} S_{2}(n, m ; x, y) \frac{t^{n}}{n !} .
$$

For $x=y=0$ in $(4.1), S_{2}(n, m)=S_{2}(n, m ; 0,0)$ are called the Stirling numbers of the second kind (see $[4,9,26]$ ).

We give some relations and properties belonging to the Hermite-based Stirling polynomials of the second kind by the following consecutive Theorems.

Theorem 4.1. We have

$$
S_{2}(n, m ; x, y)=\sum_{l=0}^{n}\left(\begin{array}{l}
n \\
l
\end{array}\right) S_{2}(l, m) H_{n-l}(x, y),
$$




$$
\begin{aligned}
& S_{2}(n, m ; x, y)=\sum_{l=0}^{n}\left(\begin{array}{l}
n \\
l
\end{array}\right) S_{2}(l, m ; 0, y) x^{n-l}, \\
& S_{2}(n, m ; x, y)=\sum_{l=0}^{\left[\frac{n}{2}\right]}\left(\begin{array}{l}
n \\
l
\end{array}\right) S_{2}(l, m ; x, 0) \frac{n ! y}{(n-2) !} .
\end{aligned}
$$

Theorem 4.2. (Derivative Properties) Each of the following formula hold true:

and

$$
\frac{\delta}{\delta x} S_{2}(n, m ; x, y)=n S_{2}(n-1, m ; x, y)
$$

$$
\frac{\delta}{\delta y} S_{2}(n, m ; x, y)=n(n-1) S_{2}(n-2, m ; x, y)
$$

Theorem 4.3. (Integral Representations) The following equalities holds true:

$$
\int_{r}^{s} S_{2}(n, m ; x, y) d x=\frac{S_{2}(n+1, m ; s, y)-S_{2}(n+1, m ; r, y)}{n+1}
$$

and

$$
\int_{v}^{\mu} S_{2}(n, m ; x, y) d y=\frac{S_{2}(n+1, m ; x, \mu)-S_{2}(n+1, m ; x, v)}{(n+1)^{2}} .
$$

Theorem 4.4. For $n, k \in \mathbb{Z}, n \geq 0,0 \leq q<1$, we have

$$
{ }_{H} B_{n, q}^{(k)}(x, y)=\sum_{a=0}^{n} \sum_{l=1}^{a+1}(-1)^{l+a+1} \frac{l !}{[l]_{q}^{k}} \frac{S_{2}(a+1, l)}{(a+1)}{ }_{H} B_{n-a}(x, y) \text {. }
$$

Proof. From (2.1), we have

$$
\begin{gathered}
\sum_{n=0}^{\infty} H_{H, q}^{(k)}(x, y) \frac{t^{n}}{n !}=\frac{\operatorname{Li}_{k, q}\left(1-e^{-t}\right)}{e^{t}-1} e^{x t+y t^{2}} \\
=\sum_{n=0}^{\infty} \frac{\left(1-e^{-t}\right)^{l}}{[l]_{q}^{k}} \frac{e^{x t+y t^{2}}}{e^{t}-1}=\sum_{n=1}^{\infty} \frac{\left(e^{-t-1}\right)^{l}}{[l]_{q}^{k}} \frac{e^{x t+y t^{2}}}{e^{t}-1} \\
=\sum_{n=1}^{\infty} \sum_{l=1}^{n} \frac{(-1)^{l+n}}{[l]_{q}^{k}} l ! S_{2}(n, l) \frac{t^{n}}{n !} \frac{e^{x t+y t^{2}}}{e^{t}-1} \\
=\sum_{a=0}^{\infty}\left(\sum_{l=1}^{a+1}(-1)^{l+a+1} \frac{l !}{[l]_{q}^{k}} \frac{S_{2}(a+1, l)}{(a+1)}\right) \frac{t^{a}}{a !}\left(\sum_{n=0}^{\infty} B_{n}(x, y) \frac{t^{n}}{n !}\right) .
\end{gathered}
$$

Replacing $n$ by $n-a$ in above equation, we get

$$
=\sum_{n=0}^{\infty}\left(\sum_{a=0}^{n} \sum_{l=1}^{a+1}(-1)^{l+a+1} \frac{l !}{[l]_{q}^{k}} \frac{S_{2}(a+1, l)}{(a+1)} H B_{n-a}(x, y)\right) \frac{t^{n}}{n !} .
$$

Comparing the coefficients of $\frac{t^{n}}{n !}$ in above equation, we get (4.2). 
Theorem 4.5. For $n, k \in \mathbb{Z}, n \geq 0,0 \leq q<1$, we have

$$
{ }_{H} B_{n, q}^{(k)}(x+u, y)=\sum_{l=0}^{\infty} \sum_{a=l}^{n}\left(\begin{array}{l}
n \\
a
\end{array}\right)(u)_{l} S_{2}(a, l)_{H} B_{n-a, q}^{(k)}(x, y) .
$$

Proof. Replacing $x$ by $x+u$ in (2.1), we have

$$
\begin{gathered}
\frac{\operatorname{Li}_{k, q}\left(1-e^{-t}\right)}{e^{t}-1} e^{(x+u) t+y t^{2}}=\sum_{n=0}^{\infty} H_{H, q}^{(k)}(x+u, y) \frac{t^{n}}{n !} \\
=\frac{\operatorname{Li}_{k, q}\left(1-e^{-t}\right)}{e^{t}-1} e^{x t+y t^{2}} \sum_{l=0}^{\infty}(u)_{l} \frac{\left(e^{t}-1\right)^{l}}{l !} \\
=\sum_{n=0}^{\infty} H B_{n, q}^{(k)}(x, y) \frac{t^{n}}{n !} \sum_{l=0}^{\infty}(u)_{l} \sum_{a=0}^{\infty} S_{2}(a, l) \frac{t^{a}}{a !} \\
=\sum_{n=0}^{\infty}\left(\sum_{l=0}^{\infty} \sum_{a=l}^{n}\left(\begin{array}{c}
n \\
a
\end{array}\right)(u)_{l} S_{2}(a, l)_{H} B_{n-a, q}^{(k)}(x, y)\right) \frac{t^{n}}{n !} .
\end{gathered}
$$

On comparing the coefficients of $\frac{t^{n}}{n !}$ in both sides, we get at the desired result (4.3).

Theorem 4.6. For $n \geq 1, n, k \in \mathbb{Z}$ and $0 \leq q<1$, we have

$$
\begin{gathered}
H_{n, q}^{(k)}(x+1, y)-{ }_{H} B_{n, q}^{(k)}(x, y)= \\
=\sum_{r=1}^{n}\left(\begin{array}{l}
n \\
r
\end{array}\right) \times\left(\sum_{l=0}^{r-1} \frac{(-1)^{r+l+1}(l+1) !}{[l+1]_{q}^{k}} S_{2}(r, l+1) H_{n-r}(x, y)\right) .
\end{gathered}
$$

Proof. Using the definition (2.1), we have

$$
\begin{gathered}
\sum_{n=0}^{\infty} H_{n, q}^{(k)}(x+1, y) \frac{t^{n}}{n !}-\sum_{n=0}^{\infty} H B_{n, q}^{(k)}(x, y) \frac{t^{n}}{n !} \\
=\frac{\operatorname{Li}_{k, q}\left(1-e^{-t}\right)}{e^{t}-1} e^{(x+1) t+y t^{2}}-\frac{\operatorname{Li}_{k, q}\left(1-e^{-t}\right)}{e^{t}-1} e^{x t+y t^{2}}, \\
=\operatorname{Li}_{k, q}\left(1-e^{-t}\right) e^{x t+y t^{2}}, \\
=\sum_{r=0}^{\infty}\left(\sum_{l=0}^{r-1} \frac{(-1)^{r+l+1}(l+1) !}{[l+1]_{q}^{k}} S_{2}(r, l+1)\right) \frac{t^{r}}{r !} e^{x t+y t^{2}}, \\
=\sum_{n=0}^{\infty}\left(\sum_{r=1}^{n}\left(\begin{array}{l}
n \\
r
\end{array}\right)\left(\sum_{l=0}^{r-1} \frac{(-1)^{r+l+1}(l+1) !}{[l+1]_{q}^{k}} S_{2}(r, l+1) H_{n-r}(x, y)\right)\right) \frac{t^{n}}{n !} .
\end{gathered}
$$

On comparing the coefficients of $\frac{t^{n}}{n !}$ on either side, we get the result (4.4). 
Theorem 4.7. For $d \in \mathbb{N}$ with $d \equiv 1(\bmod 2)$, we have

$$
\begin{aligned}
{ }_{H} B_{n, q}^{(k)}(x, y)=\sum_{p=0}^{n}\left(\begin{array}{l}
n \\
p
\end{array}\right) & d^{n-p-1} \sum_{l=0}^{p+1} \sum_{a=0}^{d-1} \frac{(-1)^{l+p+1} l ! S_{2}(p+1, l)}{[l]_{q}^{k}}(-1)^{a} \\
& \times{ }_{H} B_{n-p}\left(\frac{a+x}{d}, y\right) .
\end{aligned}
$$

Proof. Equation (2.1) can be written as

$$
\begin{aligned}
& \sum_{n=0}^{\infty}{ }_{H} B_{n, q}^{(k)}(x, y) \frac{t^{n}}{n !}=\frac{\operatorname{Li}_{k, q}\left(1-e^{-t}\right)}{e^{t}-1} e^{x t+y t^{2}} \\
= & \left(\frac{\operatorname{Li}_{k, q}\left(1-e^{-t}\right)}{t}\right)\left(\frac{t}{e^{d t}-1} \sum_{a=0}^{d-1}(-1)^{a} e^{(a+x) t+y t^{2}}\right), \\
= & \left(\sum_{p=0}^{\infty}\left(\sum_{l=1}^{p+1} \frac{(-1)^{l+p+1}}{[l]_{q}^{k}} l ! \frac{S_{2}(p+1, l)}{p+1}\right) \frac{t^{p}}{p !}\right) \\
& \times\left(\sum_{m=0}^{\infty} d^{m-1} \sum_{a=0}^{d-1}(-1)^{a}{ }_{H} B_{n}\left(\frac{a+x}{d}, y\right) \frac{t^{n}}{n !}\right) .
\end{aligned}
$$

Replacing $n$ by $n-p$ in the R.H.S. of above equation and comparing the coefficient of $\frac{t^{n}}{n !}$ on either side, we get the result (4.5).

Theorem 4.8. For $n \geq 1, n, k \in \mathbb{Z}$ and $0 \leq q<1$, we have

$$
{ }_{H} B_{n, q}^{(k)}(x, y)=\sum_{m=0}^{n} \frac{(-1)^{m+n} m !}{[m+1]_{q}^{k}} S_{2}(n, m ; x, y) .
$$

Proof. By using (2.1) and (4.1), we have

$$
\begin{gathered}
\sum_{n=0}^{\infty}{ }_{H} B_{n, q}^{(k)}(x, y) \frac{t^{n}}{n !}=\frac{\operatorname{Li}_{k, q}\left(1-e^{-t}\right)}{e^{t}-1} e^{x t+y t^{2}} \\
=\sum_{m=0}^{\infty} \frac{\left(1-e^{-t}\right)^{m}}{[m+1]_{q}^{k}} e^{x t+y t^{2}}=\sum_{n=0}^{\infty} \frac{(-1)^{m+n} m !}{[m+1]_{q}^{k}} \sum_{n=m}^{\infty} S_{2}(n, m ; x, y) \frac{t^{n}}{n !} \\
=\sum_{n=0}^{\infty}\left(\sum_{m=0}^{n} \frac{(-1)^{m+n} m !}{[m+1]_{q}^{k}} S_{2}(n, m ; x, y)\right) \frac{t^{n}}{n !} .
\end{gathered}
$$

On comparing the coefficients of $\frac{t^{n}}{n !}$ in both sides, we arrive at the desired result (4.6). 


\section{Symmetric identities}

In this section, we give general symmetry identities for the $q$-poly-Bernoulli polynomials $B_{n, q}^{(k)}(x)$ and the Hermite poly-Bernoulli polynomials ${ }_{H} B_{n, q}^{(k)}(x, y)$ with $q$ parameter by applying the generating function (2.1) and (2.2). The results extend some known identities of Khan [11-14], Pathan and Khan [20$25]$.

Theorem 5.1. Let $a, b>0$ and $a \neq b$, the following identity holds true:

$$
\begin{aligned}
& \sum_{m=0}^{n}\left(\begin{array}{c}
n \\
m
\end{array}\right) b^{m} a^{n-m} B_{n-m, q}^{(k)}\left(b x, b^{2} y\right)_{H} B_{m, q}^{(k)}\left(a x, a^{2} y\right) \\
= & \sum_{m=0}^{n}\left(\begin{array}{c}
n \\
m
\end{array}\right) a^{m} b^{n-m}{ }_{H}^{(k)} B_{n-m, q}\left(a x, a^{2} y\right)_{H} B_{m, q}^{(k)}\left(b x, b^{2} y\right) .
\end{aligned}
$$

Proof. Start with

$$
G(t)=\left(\frac{\left(\operatorname{Li}_{k, q}\left(1-e^{-a t}\right)\right)\left(\operatorname{Li}_{k, q}\left(1-e^{-b t}\right)\right)}{\left(e^{a t}-1\right)\left(e^{b t}-1\right)}\right) e^{a b x t+a^{2} b^{2} y t^{2}} .
$$

Then the expression for $G(t)$ is symmetric in $a$ and $b$ and we can expand $G(t)$ into series in two ways to obtain:

$$
\begin{gathered}
G(t)=\sum_{n=0}^{\infty}{ }_{H} B_{n, q}^{(k)}\left(b x, b^{2} y\right) \frac{(a t)^{n}}{n !} \sum_{m=0}^{\infty} H_{H, q}^{(k)}\left(a x, a^{2} y\right) \frac{(b t)^{m}}{m !} \\
\left.=\sum_{n=0}^{\infty}\left(\sum_{m=0}^{n}\left(\begin{array}{c}
n \\
m
\end{array}\right)\right) a^{n-m} b^{m}{ }_{H} B_{n-m, q}^{(k)}\left(b x, b^{2} y\right)_{H} B_{m, q}^{(k)}\left(a x, a^{2} y\right)\right) \frac{t^{n}}{n !} .
\end{gathered}
$$

On the similar lines, we can show that

$$
\begin{gathered}
G(t)=\sum_{n=0}^{\infty}{ }_{H} B_{n, q}^{(k)}\left(a x, a^{2} y\right) \frac{(b t)^{n}}{n !} \sum_{m=0}^{\infty}{ }_{H} B_{m, q}^{(k)}\left(b x, b^{2} y\right) \frac{(a t)^{m}}{m !} \\
=\sum_{n=0}^{\infty}\left(\sum_{m=0}^{n}\left(\begin{array}{c}
n \\
m
\end{array}\right) a^{m} b^{n-m}{ }_{H} B_{n-m, q}^{(k)}\left(a x, a^{2} y\right)_{H} B_{m, q}^{(k)}\left(b x, b^{2} y\right)\right) \frac{t^{n}}{n !} .
\end{gathered}
$$

Comparing the coefficients of $\frac{t^{n}}{n !}$ on the right hand sides of the last two equations, we arrive at the desired result (5.1).

Remark 5.1. On setting $b=1$ in Theorem 5.1, we get the following corollary.

Corollary 5.1. For $n, k \in \mathbb{Z}$ and $n \geq 0$, the following identity holds true:

$$
\sum_{m=0}^{n}\left(\begin{array}{c}
n \\
m
\end{array}\right) a^{n-m}{ }_{H} B_{n-m, q}^{(k)}(x, y)_{H} B_{m, q}^{(k)}\left(a x, a^{2} y\right)
$$




$$
=\sum_{m=0}^{n}\left(\begin{array}{c}
n \\
m
\end{array}\right) a^{m}{ }_{H} B_{n-m, q}^{(k)}\left(a x, a^{2} y\right)_{H} B_{m, q}^{(k)}(x, y) .
$$

Theorem 5.2. Let $a, b>0$ and $a \neq b$, the following identity holds true:

$$
\begin{aligned}
& \sum_{m=0}^{n}\left(\begin{array}{l}
n \\
m
\end{array}\right) \sum_{i=0}^{a-1} \sum_{j=0}^{b-1} H B_{n-m, q}^{(k)}\left(b x+\frac{b}{a} i+j, b^{2} z\right) B_{m, q}^{(k)}(a y) b^{m} a^{n-m} \\
= & \sum_{m=0}^{n}\left(\begin{array}{l}
n \\
m
\end{array}\right) \sum_{i=0}^{b-1} \sum_{j=0}^{a-1} H B_{n-m, q}^{(k)}\left(a x+\frac{a}{b} i+j, a^{2} z\right) B_{m, q}^{(k)}(b y) a^{m} b^{n-m} .
\end{aligned}
$$

Proof. Let

$$
\begin{aligned}
& H(t)=\left(\frac{\left(\operatorname{Li}_{k, q}\left(1-e^{-a t}\right)\right)\left(\operatorname{Li}_{k, q}\left(1-e^{-b t}\right)\right)\left(e^{a b t}-1\right)^{2}}{\left(e^{a t}-1\right)^{2}\left(e^{b t}-1\right)^{2}}\right) e^{a b(x+y) t+a^{2} b^{2} z t^{2}} . \\
& H(t)=\left(\frac{\operatorname{Li}_{k, q}\left(1-e^{-a t}\right)}{e^{a t}-1}\right) e^{a b x t+a^{2} b^{2} z t^{2}}\left(\frac{e^{a b t}-1}{e^{b t}-1}\right)\left(\frac{\operatorname{Li}_{k, q}\left(1-e^{-b t}\right)}{e^{b t}-1}\right) \\
& \times e^{a b y t}\left(\frac{e^{a b t}-1}{e^{a t}-1}\right) \\
& =\left(\frac{\operatorname{Li}_{k, q}\left(1-e^{-a t}\right)}{e^{a t}-1}\right) e^{a b x t+a^{2} b^{2} z t^{2}} \sum_{i=0}^{a-1} e^{b t i}\left(\frac{\operatorname{Li}_{k, q}\left(1-e^{-b t}\right)}{e^{b t}-1}\right) \\
& \times e^{a b y t} \sum_{j=0}^{b-1} e^{a t j} \\
& =\left(\frac{\operatorname{Li}_{k, q}\left(1-e^{-a t}\right)}{e^{a t}-1}\right) e^{a^{2} b^{2} z t^{2}} \sum_{i=0}^{a-1} \sum_{j=0}^{b-1} e^{\left(b x+\frac{b}{a} i+j\right) a t} \sum_{m=0}^{\infty} B_{m, q}^{(k)}(a y) \frac{(b t)^{m}}{m !} \\
& =\sum_{n=0}^{\infty} \sum_{i=0}^{a-1} \sum_{j=0}^{b-1} H_{n, q}^{(k)}\left(b x+\frac{b}{a} i+j, b^{2} z\right) \frac{(a t)^{n}}{n !} \sum_{m=0}^{\infty} B_{m, q}^{(k)}(a y) \frac{(b t)^{m}}{m !} \\
& H(t)=\sum_{n=0}^{\infty} \sum_{m=0}^{n}\left(\begin{array}{l}
n \\
m
\end{array}\right) \sum_{i=0}^{a-1} \sum_{j=0}^{b-1} H_{n-m, q}^{(k)}\left(b x+\frac{b}{a} i+j, b^{2} z\right) \\
& \times B_{m, q}^{(k)}(a y) b^{m} a^{n-m} \frac{t^{n}}{n !} .
\end{aligned}
$$

In similar method, we can be written as

$$
\begin{gathered}
H(t)=\sum_{n=0}^{\infty} \sum_{m=0}^{n}\left(\begin{array}{l}
n \\
m
\end{array}\right) \sum_{i=0}^{b-1} \sum_{j=0}^{a-1} H B_{n-m, q}^{(k)}\left(a x+\frac{a}{b} i+j, a^{2} z\right) \\
\times B_{m, q}^{(k)}(b y) a^{m} b^{n-m} \frac{t^{n}}{n !} .
\end{gathered}
$$


By comparing the coefficients of $\frac{t^{n}}{n !}$ on the right hand sides of the last two equations, we arrive at the desired result (5.2).

Theorem 5.3. For each pair of integers $a$ and $b$ and all integers $n \geq 0$, the following identity holds true:

$$
\begin{aligned}
& \sum_{m=0}^{n}\left(\begin{array}{c}
n \\
m
\end{array}\right) a^{n-m} b^{m} \sum_{i=0}^{a-1} \sum_{j=0}^{b-1} H_{n-m, q}^{(k)}\left(b x+\frac{b}{a} i, b^{2} z\right) B_{m, q}^{(k)}\left(a y+\frac{a}{b} j\right) \\
= & \sum_{m=0}^{n}\left(\begin{array}{c}
n \\
m
\end{array}\right) b^{n-m} a^{m} \sum_{i=0}^{b-1} \sum_{j=0}^{a-1} H B_{n-m, q}^{(k)}\left(a x+\frac{a}{b} i, a^{2} z\right) B_{m, q}^{(k)}\left(b y+\frac{b}{a} j\right) .
\end{aligned}
$$

Proof. The proof is analogous to Theorem 5.2 but we need to write equation (5.3) in the form

$$
\begin{aligned}
H(t)= & \sum_{n=0}^{\infty} \sum_{i=0}^{a-1} \sum_{j=0}^{b-1}{ }_{H} B_{n, q}^{(k)}\left(b x+\frac{b}{a} i, b^{2} z\right) \frac{(a t)^{n}}{n !} \\
& \times \sum_{m=0}^{\infty} B_{m, q}^{(k)}\left(a y+\frac{a}{b} j\right) \frac{(b t)^{m}}{m !} .
\end{aligned}
$$

On the other hand equation (5.3) can be shown equal to

$$
\begin{aligned}
H(t)= & \sum_{n=0}^{\infty} \sum_{i=0}^{b-1} \sum_{j=0}^{a-1}{ }_{H} B_{n, q}^{(k)}\left(a x+\frac{a}{b} i, a^{2} z\right) \frac{(b t)^{n}}{n !} \\
& \times \sum_{m=0}^{\infty} B_{m, q}^{(k)}\left(b y+\frac{b}{a} j\right) \frac{(a t)^{m}}{m !} .
\end{aligned}
$$

Next making change of index and by equating the coefficients of $t$ to zero in (5.5) and (5.6), we get the result (5.4).

Remark 5.2. By setting $y=0$ in Theorem 5.3, we get the following corollary.

Corollary 5.2. Let $a, b>0$ and $a \neq b$, the following identity holds true:

$$
\begin{aligned}
& \sum_{m=0}^{n}\left(\begin{array}{c}
n \\
m
\end{array}\right) a^{n-m} b^{m} \sum_{i=0}^{a-1} \sum_{j=0}^{b-1} H_{n-m, q}^{(k)}\left(b x+\frac{b}{a} i, b^{2} z\right) B_{m, q}^{(k)}\left(\frac{a}{b} j\right) \\
= & \sum_{m=0}^{n}\left(\begin{array}{c}
n \\
m
\end{array}\right) b^{n-m} a^{m} \sum_{i=0}^{b-1} \sum_{j=0}^{a-1} H_{n-m, q}^{(k)}\left(a x+\frac{a}{b} i, a^{2} z\right) B_{m, q}^{(k)}\left(\frac{b}{a} j\right) .
\end{aligned}
$$

\section{ACKNOWLEDGEMENT}

The authors are thankful to the referee for his/her valuable comments and observations which helped in improving the paper. 


\section{REFERENCES}

[1] L. C. Andrews, Special functions for engineers and mathematicians, Macmillan Co. New York, 1985.

[2] T. M. Apostol, On the Lerch zeta function, Pacific Journal of Mathematics, 1 (1951), 161-167.

[3] E. T. Bell, Exponential polynomials, Annals of Mathematics, 35 (1934), 258-277.

[4] M. Cenkcia, T. Komatsu, Poly-Bernoulli numbers and polynomials with a $q$ parameter, Journal of Number Theory, 152 (2015), 38-54.

[5] N. P. Cakić, G. V. Milonvanović, On generalized Stirling numbers and polynomials, Mathematica Balkanica, 18 (3-4) (2004), 241-248.

[6] N. P. Cakić, B. S. El-Desouky, G. V. Milonvanović, Explicit formulas and combinatorial identities for generalized Stirling numbers, Mediterranean Journal of Mathematics, 10 (2013), 57-72.

[7] G. B. Dordević, G. V. Milonvanović, Special clasees of polynomials, University of Niš, Faculty of Technology, Leskovac, 2014.

Rendiconti di Mathematica, 19 (1999), 385-391.

[8] K. W. Hwang, B. R. Nam, N. S. Jung, A note on q-analogue of poly-Bernoulli numbers and polynomials, Journal of Applied Mathematics and Informatics, 35 (5-6) (2017), 611-621.

[9] M. Kaneko, Poly-Bernoulli numbers, Journal de Théorie des Nombres de Bordeaux, 9 (1) (1997), 221-228.

[10] W. A. Khan, Some properties of the generalized Apostol type Hermite-Based polynomials, Kyungpook Mathematical Journal, 55 (2015), 597-614.

[11] W. A. Khan, A note on Hermite-based poly-Euler and multi poly-Euler polynomials, Palestine Journal of Mathematics, 5 (1) (2016), 17-26.

[12] W. A. Khan, A note on degenerate Hermite poly-Bernoulli numbers and polynomials, Journal of Classical Analysis, 2016, 65-76.

[13] W. A. Khan, I. A. Khan, S. Zia, Some properties of Hermite poly-Bernoulli polynomials, Applied Mathematics \& Information Sciences, 7 (2016), 1-7.

[14] T. Kim, Y. S. Jang, J. J. Seo, A note on poly-Genocchi numbers and polynomials, Applied Mathematical Sciences, 8 (2014), 4475-4781.

[15] T. Kim, Some identities for the Bernoulli, the Euler and the Genocchi numbers and polynomials, Advanced Studies in Contemporary Mathematics, 20 (1) (2010), 23-28.

[16] T. Kim, Y. S. Jang, J. J. Seo, Poly-Bernoulli polynomials and their applications, International Journal of Mathematical Analysis, 8 (2014), 1495-1503.

[17] T. Kim, H. K. Kwon, S. H. Lee, J. J. Seo, A note on poly-Bernoulli numbers and polynomials of the second kind, Advances in Difference Equations, 2014 (2014), 2014 219 .

[18] D. S. Kim, T. Kim, A note on ply-Bernoulli and higher order poly-Bernoulli polynomials, Russian Journal of Mathematical Physics, 22 (1) (2015), 26-33. 
[19] M. A. Pathan, W. A. Khan, Some implicit summation formulas and symmetric identities for the generalized Hermite-based polynomials, Acta Universitatis Apulensis, 39 (2014), 113-136.

[20] M. A. Pathan, W. A. Khan, Some implicit summation formulas and symmetric identities for the generalized Hermite-Bernoulli polynomials, Mediterranean Journal of Mathematics, 12 (2015), 679-695.

[21] M. A. Pathan, W. A. Khan, A new class of generalized polynomials associated with Hermite and Euler polynomials, Mediterranean Journal of Mathematics, 13 (2016), 913-928.

[22] M. A. Pathan, W. A. Khan, Some implicit summation formulas and symmetric identities for the generalized Hermite-Euler polynomials, East-West Journal of Mathematics, 16 (1) (2014), 92-109.

[23] M. A. Pathan, W. A. Khan, A new class of generalized polynomials associated with Hermite and Bernoulli polynomials, Le Matematiche, LXX (2015), 53-70.

[24] M. A. Pathan, W. A. Khan, Some new classes of generalized Hermite-based ApostolEuler and Apostol-Genocchi polynomials, Fasciculi Mathematici, 55 (2015), 153-170.

[25] C. S. Ryoo, On degenerate q-tangent polynomials of higher order, Journal of Applied Mathematics and Informatics, 35 (2017), 113-120.

[26] H. M. Srivastava, H. L. Manocha, A treatise on generating functions Ellis Horwood Limited, New York, 1984.

Waseem A. Khan

Department of Mathematics

Faculty of Science, Integral University

LUCKNOW-226026

INDIA

E-mail address: waseem08_khan@rediffmail.com

IDREES A. KHAN

Department of Mathematics

Faculty of Science, Integral University

LUCKNOW-226026

INDIA

E-mail address: khanidrees077@gmail.com

Musharraf Ali

Department of Mathematics

G.F. College

ShaHJAHANPUR-242001

INDIA

E-mail address: drmusharrafali@gmail.com 